\title{
Library Book Recommendation System of Tianjin University of Technology
}

\author{
Zishuai Yang 1, a, Yingchun Wang ${ }^{1, b}$, Yanquan Liü, c \\ ${ }^{1}$ School of Tianjin, Technology University, Tianjin 300000, China; \\ 2 School of America, Southern Connecticut State University (New Haven). \\ a562125116@qq.com, b13512408866@126.com, cliuy1@southernct.edu
}

\begin{abstract}
This paper makes a systematic and in-depth study on the functional module design of the book recommendation system in the library of Tianjin University of Technology. In order to ensure the standardization and scientificalness of the research and development process and meet the needs of library use, the research and field investigation methods are comprehensively used, and the recommendation system theory and methods are used for reference to understand the relevant recommendation algorithms and strictly standardize the criteria for consideration thus completing the design of the functional module of the book recommendation system in the library of Tianjin University of Technology.
\end{abstract}

Keywords: Library; Personalized recommendation; Recommendation algorithm; Efficient.

\section{Introduction}

This topic intends to analyze, design, and implement a personalized book recommendation system for university libraries. Function: Implement different book functions for different users; implement user login information management, borrow function management; book basic information management; book label function management; book comment function management; search function management. Performance: Improve the responsiveness of the system with a new architecture.

This system can be deployed in the university library, so that the library can make efficient use of the rich collection resources and enrich the learning life of the majority of teachers and students. For students, it provides a more convenient means of obtaining book information.

In 1979, Schumann, an American scholar, put forward the idea that " libraries and information providers should provide new services to meet personal needs", which can be said to be the embryonic form of the idea of personalized information services abroad [1]. The recommendation system is a special form of information filtering system. By analyzing the user's historical interest and preference information, the recommendation system can determine the items that the user may like now and in the future in the project space, and then actively provide corresponding project recommendation services to the user [2].

\section{System Overall Design Description}

\subsection{System Modular Structure Design}

\subsubsection{Basic User Information Management Function}

The management functions of system users include the registration of user information, information verification of logged-in users, background management of administrators, and management of user rights.2.1.2 Sub heading.

\subsubsection{Library Management Function}

The book management function of the system includes the management of book information, including book warehousing, book moving and book information updating. Book Review Management: Includes users publishing book reviews and administrators deleting book reviews. Book label management: includes administrators adding book labels, deleting book labels, and 
modifying book labels. Management of book borrowing: including users borrowing books and returning borrowed books.

\subsubsection{Book Recommendation Function}

The system's book recommendation function, the focus of this paper, includes the user-based book recommendation algorithm USER CF and the item-based book recommendation algorithm ITEM CF. There is also a cold recommendation function for new users.

\subsubsection{System Search Function}

The search function of the system includes the search of books in the collection, the search of users and the search of discussion posts.

\subsubsection{Discuss the Post Management Function}

The system's discussion post functions include users publishing discussion posts, modifying discussion posts, administrators deleting non-compliant posts, users replying to discussion posts, users praising discussion posts, users collecting discussion posts, praising discussion posts, etc.

\subsection{Design of System Development Platform}

Tab.1 System development platform

\begin{tabular}{c|c}
\hline Network environment & Internet \\
Hardware platform & Tencent cloud server $\quad$ CPU:2core \\
Operating system & Memory: $2 \mathrm{G} \quad$ Hard disk space:50G \\
Database platform & Ubantu 14.04 \\
Storage platform & MySQL, Redis \\
Language environment & Tengxuyun object storageCOS \\
Development software & Python3.6, Django2.0, bootstrap3.0 \\
\hline
\end{tabular}

\subsection{Overview of System Architecture}

When a user issues an HTTP request, it will be accepted by NGINX first. NGINX will analyze which resources in the request need to be obtained dynamically and which resources can be obtained directly from static state. The content that needs to be dynamically acquired is transferred to Django without going through Django directly from statically acquired resources, and Django will handle dynamic requests, some of which are cached in the memory database Redis, and Django will first look for them from Redis, and if it finds a direct return, it will not go through MySQL. If not, go to MySQL and refresh the Redis cache and return it to the user. At the same time, some media resources return information. Such as pictures, will return the picture address of a CDN, and the user browser will actually download the picture resources to the nearest CDN node after receiving this address. $\mathrm{CDN}$ is also a national distribution node and will regularly synchronize picture resources from our picture storage.

\section{Introduction to Recommendation System}

\subsection{Web Crawler}

Web crawler, also known as web spider or web information collector, is a computer program or automated script that automatically downloads web pages and is an important part of search engines. Web crawler usually starts from a URL set called a seed set. It first places all these URLs in an orderly queue to crawl, takes out the URLs from them in a certain order and downloads the pointed pages, analyzes the page contents, extracts new URLs and stores them in the queue to crawl, thus repeating 
the above process until the URL queue is empty or certain crawling termination conditions are met, thus traversing the Web.

It is impossible to make recommendation features by directly using book names, and a feature vector needs to be constructed. Write a multi-threaded web crawler to obtain the pre-closed data from the network. The multi-threaded crawler is an efficient crawler that can play the computing potential on multi-core machines.

Specific to the implementation algorithm:

(1) Get the book name to Douban Reading (https://book.douban.com/) and find the book details page.

(2) After finding it, distribute it to the corresponding working thread.

(3) Find the tagging of the book by the top 50 users on the book details page.

(4) Statistics of corresponding keywords.

Since it is a library itself, to do a good job in information security, it involves stealing chains. Theft chain refers to an act of displaying and playing on a web page (or client) set by it by grabbing other people's content address, occupying other people's bandwidth, copyright content and other hard and software resources [3]. The object store has its own anti-theft chain to prevent web crawler from crawling and other websites from stealing the chain.

\subsection{Application Algorithm}

It mainly uses collaborative filtering algorithm, which was used to solve the information overload at the earliest. Screening news to help news viewers filter out interesting content; After being applied to Amazon's e-commerce system, it is used to recommend books that may be of interest to customers.

For item-based collaborative filtering, item cf still takes calculating similarity as its main step. According to all users' evaluation of books, the similarity between items and items is found, and then similar items are recommended to the user according to the user's historical preference information. by use that obtained key word vector of the book, the association strength between the books can be calculated and the item cf can be made.

Specific to the implementation algorithm:

(1) The relationship between each book borrowed by the user in the past and all other books is calculated.

(2) Sort related books from high to low according to the degree of relationship.

(3) Add the top 10\% keywords to the user keyword feature vector.

User-based collaborative filtering, User CF discovers features that users do not show through lending activities by discovering people with similar characteristics to users. The user's borrowing record is regarded as an $\mathrm{N}$-dimensional vector, and then users with the same hobbies and habits are obtained by using statistical tools.

Specific to the implementation algorithm:

(1) Each person and all other people do relationship calculation.

(2) Rank the related people from high to low according to the degree of relationship.

(3) Take the top $10 \%$.

(4) Do the difference operation between the keyword set of these people and the keyword set of the user. Update to the corresponding user's extended keyword vector.

When recommending, select part of the keywords obtained from Item CF and select part of the keywords obtained from User CF. It is now 4:1. Add the book corresponding to the keyword to the recommended collection.

\subsection{Cold Recommendation}

Cold recommendation refers to the recommendation of users without historical data. For university libraries, through the analysis of historical data, users without historical data can be labeled with appropriate manual rules, specifically:

(1) Labeling freshmen with high numbers and four grades, and labeling junior students with one's deceased father grind and employment. 
(2) label the students' major, such as the students majoring in computer science and technology.

(3) Label popular books with a wide range of reading, such as novels and inspirations.

\section{Summary}

Recommendation system is a lubricant that can improve the system. The development of this system can fill the blank of personalized book recommendation in university libraries. Let the library's book resources serve users better. From the beginning of design to landing practice, the development of this system proves the feasibility of the recommendation system in the field of university libraries. With the progress of the times, more algorithms will be applied in the library in the future. Through the development of this system, no recommendation system has been made. It also integrates cloud computing technology and studies how to build a high-performance and high-concurrency system. This is also of great reference significance to other systems in the future. Finally, when the user opens the web page, the response speed is within $1 \mathrm{~s}$ and the recommendation accuracy is above $90 \%$, achieving the set goal.

In addition to meeting the design requirements of the established management information system, this system has built a practical, accurate and recommended system, which enables the front end to display the back-end progress in real time during offline calculation through the application of Web Socket based on HTTP / 2.0. In the past, Web pages that did not do Web Socket often used Ajax polling to process real-time messages in the background. Because HTTP is based on TCP, one request requires three-way handshake, four waves, one response requires three-way handshake, four waves, and six requests as a whole. Due to the fixed waiting time of TCP, this method wastes front-end resources and increases the delay. In the Web Socket used in this system, the server can actively push messages to the front end, and the browser only maintains a TCP connection.

Since this system spans HTTP 1.0 and HTTP 2.0, it has done reverse proxy at NGINX level, and requests for different pages go through different gateway protocols.

\section{References}

[1]. Patricia Class Schuman. Library and information services for meeting Personal needs: a discussion guide. National Commission on Libraries and Information Science, 1979, p. 86-87.

[2]. Shitao Xu. Research on Probabilistic Matrix Factorization Based Recommendation Algorithm (Master's degree, Shanghai Jiao Tong University, China 2014). p.1-2.

[3]. Changjun Lv. A Brief Analysis of Deep Links, Framed Links and Theft Chains-—From the Perspective of Information Network Transmission Rights. Chinese copyright. (2016) No. 02, p. 39-42. 